\title{
SOME PRINCIPLES GOVERNING THE CHOICE AND UTILIZATION OF PERMANENT-MAGNET STEELS
}

\author{
By Raymond L. Sanford
}

\section{ABSTRACT}

The addition of substantial percentages of cobalt to the composition of permanent-magnet steels leads to markedly superior qualities. The cost of cobalt steels may be several times that of the older types, however, and their use is warranted only under certain circumstances. Criteria for the comparison of the quality of magnet steels are discussed, and the conditions under which the use of the more expensive steels is justified are outlined.

\section{CONTENTS}

I. Introduction

II. The demagnetization curve

III. The hyperbolic law and the reduced demagnetization curve....

IV. Comparison of magnet steels

V. Stabilization by partial demagnetization

\section{INTRODUCTION}

Since the discovery by Honda ${ }^{1}$ of the beneficial effects of the addition of substantial percentages of cobalt to the composition of permanent-magnet steels, a number of types of magnet steel have been developed whose properties are markedly superior to those of the older types of tungsten and chromium steels. Very high values of coercive force have been obtained before but always accompanied by such a reduction in residual induction that the materials had no practical value. The addition of cobalt, however, brings about a large increase in coercive force without a corresponding decrease in residual induction, and if it were not for the element of cost it is probable that the cobalt steels would displace the older types to a large extent. Unfortunately, however, cobalt is relatively very expensive, and the cost of cobalt steels ranges from four to ten times as great as that of tungsten or chromium steel. The cost of working is also somewhat greater. With this fact in mind, it may be of

1 Honda and Saito, Phys. Rev., 16, p. 494; 1920.

$66622^{\circ}-27$ 
advantage to consider just what is to be gained by the use of cobalt steels and under what conditions their substitution for the older types is justified.

\section{THE DEMAGNETIZATION CURVE}

The function of a permanent magnet may be either to maintain a constant magnetic flux in an air gap, as in electrical measuring instruments, or to store energy in the magnetic field which can later be delivered to an electrical circuit associated with it, as in magnetos.

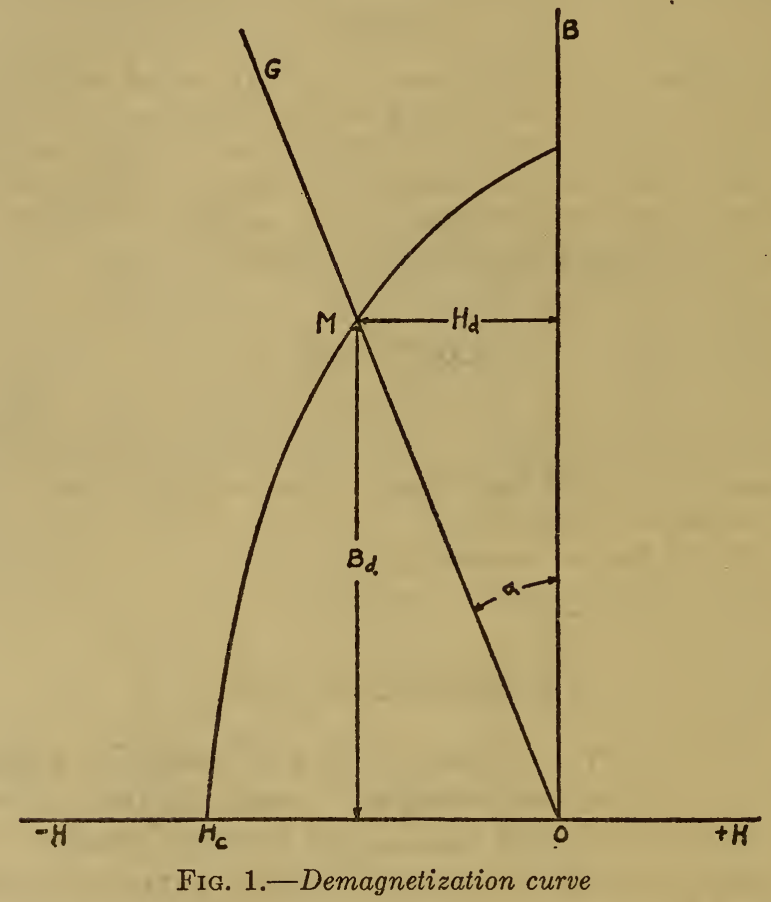

In either case the magnet also maintains a leakage flux which is not useful, but which must be taken into account in the complete design of a magnet. On account of this leakage the flux in a magnet is not uniform throughout its length but varies from a maximum in the neutral section to a minimum at the ends. For the purpose of the present discussion it will be of advantage to neglect the effect of leakage in order to avoid confusion, but it must be distinctly borne in mind that this can not be done in the case of actual design.

The designer of permanent magnets is primarily concerned with the part of the descending hysteresis curve lying between the residual induction and the coercive force, as indicated in Figure 1. This may be called the demagnetization curve. 
If we let

$H_{\mathrm{d}}=$ demagnetizing force acting on the magnet,

$B_{\mathrm{d}}=$ value of $B$ in the magnet,

$l_{\mathrm{m}}=$ length of the magnet in $\mathrm{cm}$,

$A_{\mathrm{m}}=$ cross-sectional area of magnet in $\mathrm{cm}^{2}$,

$H_{\mathrm{g}}=$ the field strength in the gap,

$l_{\mathrm{g}}=$ length of gap in $\mathrm{cm}$,

$A_{\mathrm{g}}=$ cross-sectional area of gap in $\mathrm{cm}^{2}$,

then, neglecting leakage, we have the following relations:

$$
B_{\mathrm{d}} A_{\mathrm{m}}=H_{\mathrm{g}} A_{\mathrm{g}}
$$

and

$$
H_{\mathrm{d}} l_{\mathrm{m}}=H_{\mathrm{g}} l_{\mathrm{g}}
$$

That is, the total flux in the magnet is equal to the total flux in the gap and the magnetomotive forces are also equal. The tangent of angle $a$, Figure 1 , is $\frac{H_{\mathrm{d}}}{B_{\mathrm{d}}}$, and from equations (1) and (2) its value ${ }^{2}$ is

$$
\frac{H_{\mathrm{d}}}{B_{\mathrm{d}}}=\frac{l_{\mathrm{g}} A_{\mathrm{m}}}{l_{\mathrm{m}} A_{\mathrm{g}}}
$$

Tan $a$, and consequently the induction in the steel, is fixed, therefore, not only by properties of the steel but also by the relative dimensions of the magnet and the gap. An empirical law laid down in 1889 by Hookham ${ }^{3}$ states that if satisfactory permanency is to be assured, the ratio $\frac{l_{\mathrm{g}} A_{\mathrm{m}}}{l_{\mathrm{m}} A_{\mathrm{g}}}$ should never be greater than $1 / 70$. This does not hold, however, for the cobalt steels with their larger values of coercive force.

Magnets are sometimes designed with little, if any consideration of economy in the use of material. It is possible, however, in the ideal case to determine the value of flux density which corresponds to the minimum volume of steel.

If we solve for $A_{\mathrm{m}}$ and $l_{\mathrm{m}}$ in equation (1) and (2)

and

$$
A_{\mathrm{m}}=\frac{H_{\mathrm{g}} A_{\mathrm{g}}}{B_{\mathrm{d}}}
$$

$$
l_{\mathrm{m}}=\frac{H_{\mathrm{g}} l_{\mathrm{g}}}{H_{\mathrm{d}}}
$$

and the volume of the magnet is

$$
V=A_{\mathrm{m}} l_{\mathrm{m}}=\frac{H_{\mathrm{g}}^{2} A_{\mathrm{g}} l_{\mathrm{g}}}{B_{\mathrm{d}} H_{\mathrm{d}}}
$$

When $B$ and $H$ are not plotted to the same scale, the ratio of the coordinates must be taken into account.

${ }^{3}$ Hookham: Phil. Mag., 27, p. 186; 1889. 
That is, when the product of $B_{\mathrm{d}}$ and $H_{\mathrm{d}}$ is a maximum, the volume of steel required is a minimum. In the right-hand part of Figure 2 the products of $B_{\mathrm{d}}$ and $H_{\mathrm{d}}$ are plotted as abscissas against values of $B_{\mathrm{d}}$ as ordinates, indicating the maximum value of the $B_{\mathrm{d}} H_{\mathrm{d}}$ product. In the practical case, of course, on account of the influence of leakage, the induction in the magnet is not constant throughout its length, and the best that can be done is to make the average value correspond to that at which the product is a maximum.

This relationship furnishes also the best general basis for the comparison of magnet steels, the steel having the greatest $\left(B_{\mathrm{d}} H_{\mathrm{d}}\right) \max$ requiring the least volume for a given set of requirements.

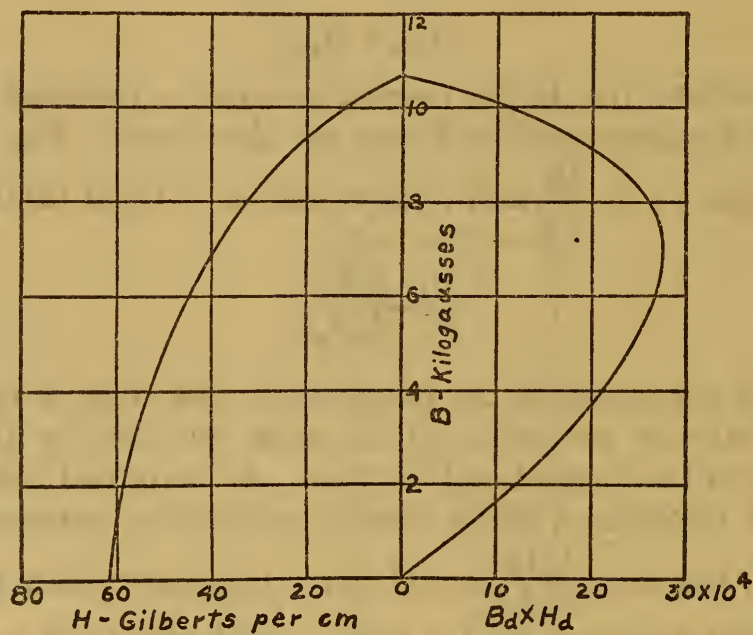

FIG. 2.-Demagnetization curve and $B H$ product

\section{THE HYPERBOLIC LAW AND THE RATIO CURVE}

Watson, ${ }^{4}$ in an exceedingly valuable article on the properties of permanent-magnet steel, states that if the material has been properly hardened so as to produce a homogeneous structure it behaves as though there were but a single magnetically distinct component and that the demagnetization curve is part of a hyperbola.

$\mathrm{He}$ also describes a graphical method for constructing the demagnetization curve, for the mathematical demonstration of which the reader is referred to the original article. The construction is illustrated in Figure 3. $\quad B_{\mathrm{s}}$ is the saturation value and $B_{\mathrm{r}}$ and $H_{\mathrm{c}}$ are the residual induction and coercive force, respectively. Horizontal and vertical lines drawn through $B_{\mathrm{s}}$ and $R H_{\mathrm{c}}$, respectively, meet at the point $S$ and are the asymptotes of the curve. $R$ is the ratio of the 
saturation value to the residual induction $\left(R=\frac{B_{\mathrm{s}}}{B_{\mathrm{r}}}\right)$. Horizontal and vertical lines are also drawn through $B_{\mathrm{r}}$ and $H_{\mathrm{c}}$, as shown. Now, if a line is drawn from $S$ intersecting the vertical through $H_{\mathrm{c}}$ and the axis of $H$, as at $B_{1}$ and $H_{1}$, respectively, horizontal and vertical lines through these intersections will meet at a point as $M_{1}$, which is a point on the curve. By a similar procedure other points on the curve can be located.

By an inverse process, the point $S$ can be located or a given curve can be tested to see whether or not it follows the hyperbolic law. If horizontal and vertical lines are drawn from observed points on a

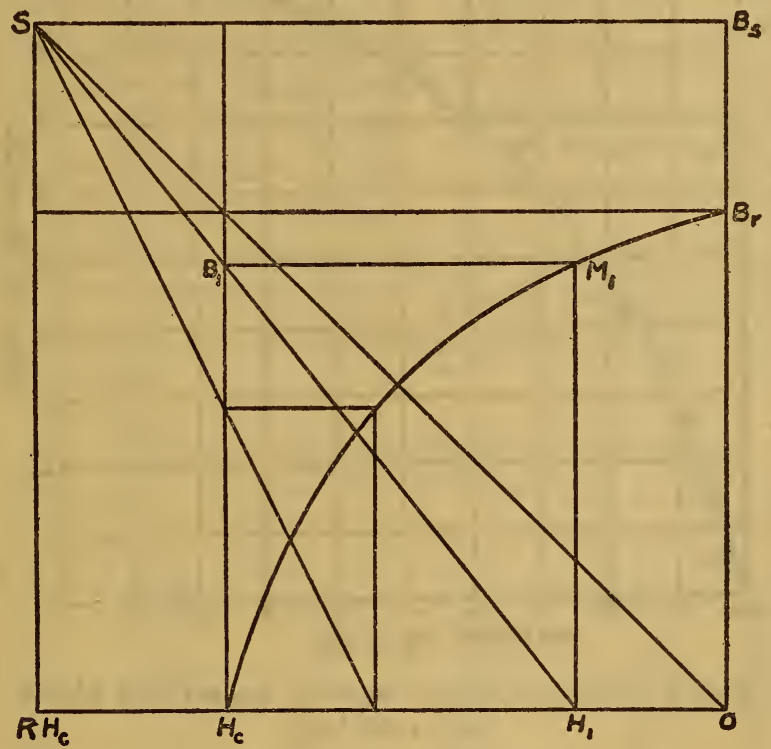

FIG. 3.-Graphical method of construction of the demagnetization curve

given curve to the vertical through $H_{\mathrm{c}}$ and the axis of $H$, the lines drawn through the corresponding intersections should meet at a single point. If they do not meet at a common point, then the curve does not follow the hyperbolic law. According to Watson, this is a good test as to whether or not the material has been properly heattreated.

During the course of the present study another way of plotting the demagnetization curve was tried, namely, the ratio of induction to the residual induction against the ratio of magnetizing force to the coercive force; that is, $B_{\mathrm{d}} / B_{\mathrm{r}} \nabla . H_{\mathrm{d}} / H_{\mathrm{c}}$. This was done for a number of steels for which data were available, and it was found that there was surprisingly little difference in the curves plotted on this basis for materials having coercive-force values ranging from 
approximately 50 to over 200 gilberts per centimeter. This is shown in Figure 4. An examination of the curves showing the greatest deviation from the average showed in each case that the curve did not follow the hyperbolic law. This leads to the interesting possibility that a single master curve, which might be called the reduced demagnetization curve, could be used to represent the characteristics of any properly hardened magnet steel, provided only that the values of residual induction and coercive force were known. Such a curve is shown in Figure 5. For this curve the ratio of saturation value

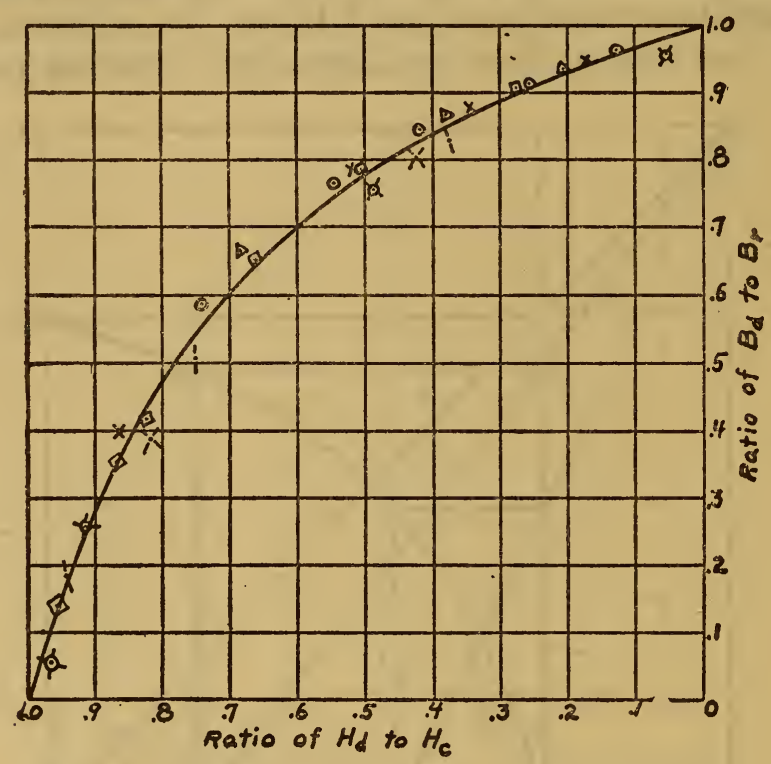

Fig. 4.-Data for various types of magnet steel plotted on a ratio basis

to residual induction $(R)$ is 1.38 , and the construction was carried out by the graphical method just outlined.

It can be shown that a line drawn from $S$ to the origin cuts the curve at the point where the $B_{\mathrm{d}} H_{\mathrm{d}}$ product is a maximum. For the ratio curve this product is a maximum when the induction is approximately 0.65 times the residual induction and the demagnetizing force is approximately 0.65 times the coercive force. If this curve represents the properties of all classes of steel, when properly hardened, then the maximum $B_{\mathrm{d}} H_{\mathrm{d}}$ product ought to be

$$
\left(B_{\mathrm{d}} H_{\mathrm{d}}\right)_{\max }=0.65 B_{\mathrm{r}} \times 0.65 H_{\mathrm{c}}=0.42 B_{\mathrm{r}} H_{\mathrm{c}}
$$

This relationship, therefore, should serve as a means of checking the validity of the reduced demagnetization curve as representing the properties of any properly hardened magnet steel. With this end in view, the data given in recent published articles and also 
many obtained in the course of tests made at the Bureau of Standards have been examined. Many of the published values are admittedly estimates, and the accuracy of experimentally determined values may be somewhat in doubt; but it was found that this ratio appears to hold within less than 10 per cent, which, in general, would be amply close for practical purposes. In Table 1 are given values taken from papers by Watson ${ }^{5}$ and Kayser ${ }^{6}$ covering materials having coercive-force values ranging from 55 to 280 gilberts per centimeter.

It is only within the last few years that the significance of the $\left(B_{\mathrm{d}} H_{\mathrm{d}}\right)_{\max }$ product has been gene-

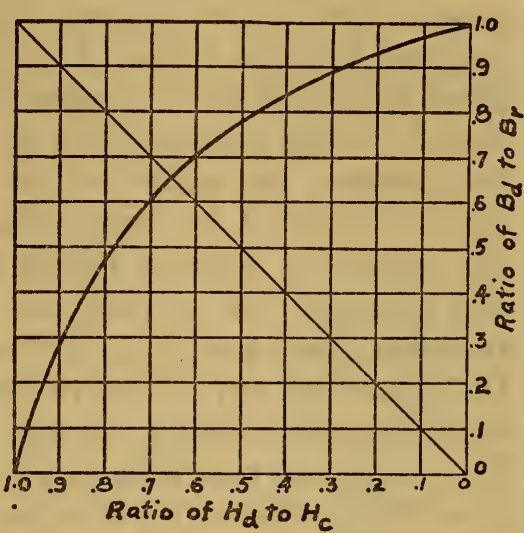

Frg. 5.-Reduced demagnetization curve rally recognized, and consequently few data have been published. It may be that when more data become available a slightly different value for $B_{\mathrm{s}} / B_{\mathrm{r}}$ will be indicated, but the value 1.38 seems to be the best at present.

TABLE 1.-Ratio of $\left(B_{\mathrm{d}} H_{\mathrm{d}}\right)_{\mathrm{max}}$ to $B_{\mathbf{r}} \times H_{\mathrm{o}}$

\begin{tabular}{|c|c|c|c|c|c|}
\hline$H_{0}$ & $B_{\mathrm{r}}$ & $B_{\mathrm{r}} \times H_{\mathrm{o}} \times 10^{-6}$ & $(B H)_{\mathrm{m} 3 \mathrm{~s}} \times 10^{-8}$ & Ratio & Reference \\
\hline & & & & \\
\hline 55 & 10,000 & 5.5 & 2.20 & 0.40 & $(1)$ \\
65 & 10,000 & 6.5 & 2.80 & .43 & $(1)$ \\
66.3 & 12,270 & 8.14 & 3.40 & .42 & $(1)$ \\
96 & 11,200 & 10.7 & 4.30 & .40 & $(2)$ \\
97 & 9,900 & 9.6 & 3.75 & .39 & $(3)$ \\
130 & 10,159 & 13.2 & 5.50 & .42 & $(3)$ \\
160 & 9,250 & 14.8 & 6.20 & .42 & $(2)$ \\
180 & 8,900 & 16.0 & 6.75 & .42 & $(2)$ \\
210 & 8,300 & 17.4 & 7.00 & .40 & $(2)$ \\
220 & 9,000 & 19.8 & 7.00 & .35 & $(1)$ \\
230 & 7,700 & 17.7 & 7.25 & .41 & $(2)$ \\
280 & 9,500 & 26.6 & 9.00 & .34 & $(1)$ \\
\hline
\end{tabular}

1 Kayser, Electrician, 90, p. 557; 1923.

Watson, Jour. Inst. Elec. Eng., 61, p. 641; 1923.

3 Watson, Engineering, 118, p. 274; 1924.

According to Watson, improper heat treatment leads to a "lean" hysteresis loop which would give a low value for the ratio of $\left(B_{\mathrm{d}} H_{\mathrm{d}}\right)_{\max }$ to $B_{\mathrm{r}} \times H_{\mathrm{c}}$. It may be significant, therefore, that the ratios which differ from 0.42 by the greatest amount are lower. Perbaps this ratio will prove to be a good criterion for the determination of the best heat treatment for steel of a given composition.

6 Watson, J. Inst. Elec. Eng., 61, p. 641; 1923; and Engineering, 118, p. $274 ; 1924$.

Kayser, Electrician, 90, p. 557; 1923. 


\section{COMPARISON OF MAGNET STEELS}

If the reduced demagnetization curve represents the properties of magnet steel to a satisfactory degree of approximation, it is necessary to know only the values of residual induction and coercive force of a given steel in order to compare its properties with those of any other steel for which the same data are known. For any point on the curve, for instance, the actual values of induction and magnetizing force can be obtained by multiplying the corresponding coordinates by the values of residual induction and coercive force, respectively. For example, let us compare the properties of two steels-A, a tungsten steel, and $B$, a cobalt steel. A has a residual induction of 10,000 gausses and a coercive force of 61 . B has a residual induction of 9,000 gausses and a coercive force of 200 gilberts per centimeter. Let us suppose that for a given air gap the length and cross-sectional area of the steel are such that $\tan \alpha=0.0003$. Since the ratio of coordinates is $B_{\mathrm{r}} / H_{\mathrm{c}}$, the actual tangent on the ratio diagram is for steel $A, 0.492$, and for steel $\mathrm{B}, 0.135$. The corresponding inductions are 8,350 and 8,640 , respectively. In other words, the cobalt steel gives an induction greater than the tungsten steel by about $31 / 2$ per cent. On the other hand, if the design had been such that $\tan \alpha=0.01$, the actual tangents for $\mathrm{A}$ and $\mathrm{B}$ would have been 1.64 and 0.45 , respectively, and the induction for steel $\mathrm{A}$ would be 4,850 and for steel B would be 7,650, an advantage of over 78 per cent in favor of the cobalt steel.

Another basis of comparison is the relative volume required to maintain the same value of flux density in a given air gap. From equation (6) the volume of a magnet is

$$
V=\frac{H_{\mathrm{g}}^{2} A_{\mathrm{g}} l_{\mathrm{g}}}{B_{\mathrm{d}} \bar{H}_{\mathrm{d}}}
$$

If $H_{\mathrm{g}}, A_{\mathrm{g}}$, and $l_{\mathrm{g}}$ are the same for both steels, the minimum volumes required will be inversely proportional to the respective values of $(B H)_{\max }$.

From equation (7) we have seen that $\left(B_{\mathrm{d}} H_{\mathrm{d}}\right)_{\max }=0.42 B_{\mathrm{r}} H_{\mathrm{c}}$, so that for steel $\mathrm{A}$

$$
\text { 1 }\left(B_{\mathrm{d}} H_{\mathrm{d}}\right)_{\max }=0.42 \times 10,000 \times 61=256,200
$$

and for steel B

$$
\left(B_{\mathrm{d}} H_{\mathrm{d}}\right)_{\text {max }}=0.42 \times 9,000 \times 200=756,000
$$

and the ratio of volumes required for the most economical design is 2.95. In other words, the same effect can be obtained with about one-third as great a volume of cobalt steel as would be required for the tungsten steel. On the other hand, if the unit cost of the cobalt steel were more than three times that of the tungsten steel, its use 
would be warranted only if consideration of weight or space were of more importance than cost or the conditions of operation were so severe that the tungsten steel would be unsatisfactory.

From the above it can be seen that the mere substitution of cobalt steel for tungsten or chromium steel without an appropriate change in design will probably lead to disappointing results, especially if the original design was well adapted to the lower grade steel.

For applications where weight or space are of primary importance and cost is a secondary consideration, however, considerable advantage is to be gained from the use of the cobalt steels. It is also evident that certain results can be obtained with cobalt steel which are utterly impossible with the older types.

The steels at present available are the well-known tungsten and chromium steels having coerciveforce values from 50 to 70 gilberts per centimeter and residual inductions from 9,000 to 11,000 gausses;

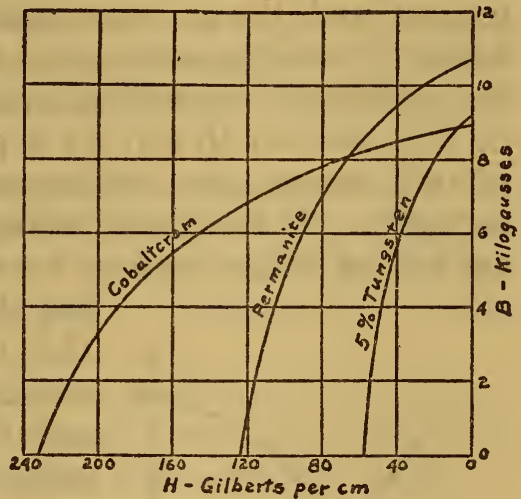

FIG. 6.-Typical demagnetization curves for three kinds of magnet steel Cobaltcrom and permanite contain approximately 35 and 15 per cent of cobalt, respectively cobalt steels having a cobalt content of the order of 15 per cent, with coercive-force values of the order of 100 gilberts per centimeter and residual inductions from 8,000 to 10,000 gausses; and cobalt steels having approximately 35 per cent cobalt which give coercive forces of over 200 gilberts per centimeter, with residual inductions from 7,500 to 9,500 gausses. Demagnetization curves for typical steels of the three classes are shown in Figure 6.

\section{STABILIZATION BY PARTIAL DEMAGNETIZATION}

A permanent magnet is subject to deterioration due to three causes-changes in internal structure, mechanical shocks, and magnetic fields. The effect of structural changes can be overcome by a suitable aging or maturing process, usually a low-temperature heat treatment, which brings about in a short time changes which would otherwise require a period of months or even years. The effect of mechanical shock can be minimized by subjecting the magnet initially to shocks of such an extent that additional jars will have no further influence. The effect of transient demagnetizing field can be guarded against to a certain degree by partial demagnetization.

The procedure can best be described by reference to Figure 7 . Suppose that the relative dimensions of the magnet and gap are such that the equilibrium point when the magnetizing force is removed 
is represented by $M_{1}$, corresponding to an induction and magnetizing force of $B_{1}$ and $H_{1}$, respectively. Now, suppose that the magnet is subjected to the influence of a demagnetizing force. The equilibrium point will move down the demagnetization curve to some point such as $M_{2}$. When the demagnetizing force is removed, the equilibrium point does not return to $M_{1}$ but rather follows a minor hysteresis loop, such as $M_{2} M_{3} B_{4}$, until the point $M_{3}$ is reached, when the magnet and the gap are again balanced. Subsequent temporary fields will send the state point of the magnet back and forth within the field of the minor loop, always leaving the magnet at some point on $O M_{1}$ between $M_{3}$ and the upper branch of the loop. As this loop is very narrow, the permanent effect of small fields is generally negligible. If the demagnetizing force exceeds $H_{2}$ in value, the magnet will no longer work on the same minor loop, but on a lower one, and the induction is permanently reduced.

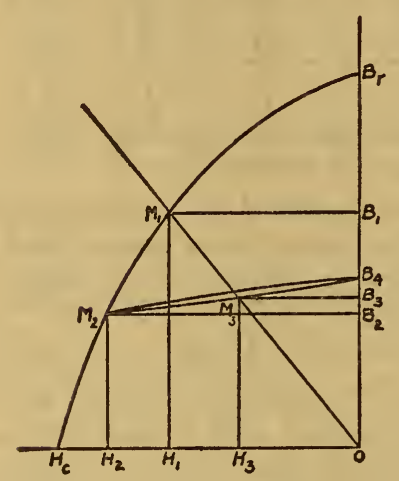

FIG. 7.-Stabilization by partial demagnetization $\mathrm{H}_{3} \mathrm{H}_{2}$ then represents the degree of magnetic stability of a magnet originally saturated and then partially demagnetized to the point $M_{2}$. The degree of partial demagnetization required for stabilization depends upon the service for which the magnet is intended and the magnitude of the demagnetizing forces it is likely to encounter.

It is easy to see that steels having high values of coercive force can be stabilized to withstand demagnetizing influences of much greater magnitude than can steels of lower coercive forces. The procedure just outlined also furnishes a means of adjusting the magnet strength to the required value, so that very exact data for the demagnetization curve are not essential for satisfactory design.

\section{SUMMARY}

To recapitulate briefly:

1. The addition of substantial percentages of cobalt to the composition of permanent-magnet steel results in the attainment of coercive-force values from two to five times as great as those of the commonly used tungsten or chromium steels without a serious reduction in residual induction.

2. On account of the relatively high cost of cobalt, the cobalt steels may be from four to ten times as expensive as the other types of steel. For this reason the element of cost is an important factor in the selection of a magnet steel for a given purpose.

3. The part of the hysteresis loop lying between the residual induction and the coercive force represents the magnetic properties of 
steel with which the designer of permanent magnets is primarily concerned. This may be called the demagnetization curve, and for properly hardened material is part of a hyperbola.

4. A graphical method can be used for constructing the hyperbolic demagnetization curve or for testing an observed curve to see if it fits the hyperbolic low.

5. If $B / B_{\mathrm{r}}$ is plotted against $H / H_{\mathrm{c}}$, it has been found that curves for all types of steel are so nearly alike that a single curve can be constructed which represents to a sufficiently close approximation the properties of any type of steel, provided only that the values of $B_{\mathrm{r}}$ and $H_{\mathrm{c}}$ are known.

6. The best general criterion of the quality of magnet steel is the maximum value of the product of $B_{\mathrm{d}}$ and $H_{\mathrm{d}}$ on the demagnetization curve, and since this appears to be directly proportional to the product of $B_{\mathrm{r}}$ by $H_{\mathrm{c}}$, the $B_{\mathrm{r}} H_{\mathrm{c}}$ product can be used in comparing the quality of various types of steel.

7. Cobalt steels are superior in quality to tungsten and chromium steels, but this superiority can only be utilized by means of appropriate design. The mere substitution of cobalt steel without change of design will usually lead to disappointing results.

8. Magnets can be stabilized against permanent deterioration from transient demagnetizing fields by partial demagnetization. The cobalt steels can be stabilized by this method to a greater degree than the steels having lower values of coercive force.

9. In general, it may be said that although by the use of cobalt steels some results can be obtained which are utterly impossible with the tungsten and chromium steels more commonly employed, their use is generally not economically justified except in cases where space or weight are of primary importance and cost is a secondary consideration or where particularly severe conditions must be met.

Washington, August 16, 1927. 